\title{
Magnitude of Undernutrition and Associated Factors among Infants and Young Children aged 6- 23 Months in Sinan district, Northwest Ethiopia Amhara regional state: A crossectional study
}

Mastewal Giza Amera ( $\sim$ mastewalgiza@gmail.com )

Woldia University https://orcid.org/0000-0002-7271-0904

Gemechu Kumera Feyira

Debre Markos University

Nurilign Abebe Moges

Debre Markos University College of Health Science

Enyew Fenta Mengistu

Debre Markos University College of Health Science

Yihenew Sewale Bizu

Debre Markos University College of Health Science

\section{Research}

Keywords: children, infant, undernutrition, young

Posted Date: January 15th, 2021

DOI: https://doi.org/10.21203/rs.3.rs-146003/v1

License: (c) (i) This work is licensed under a Creative Commons Attribution 4.0 International License.

Read Full License 


\section{Abstract}

Background: Childhood undernutrition continues to be the most serious public health problem in the developing world particularly in the rural area. Determining the nutritional status among children less than two years is needed to plan a good nutrition intervention and to provide nutrition education to the community. Despite this, there is limited research conducted in the study area.

Objective: To assess undernutrition and associated factors among infants and young children aged 6-23 months in Sinan District, Northwest Ethiopia, 2019.

Methods: A Community -based cross-sectional study was conducted among 431 child-mother pairs from March 10, 2019-April 20, 2019 in Sinan District. WHO Anthro software (version 3.2.2) was used to generate the Z-scores. Data were entered using Epi-Data version 4.2 and analysis was done using SPSS version 25. Bivariable and multivariable binary Logistic Regression were fitted. In the multivariable analysis, $\mathrm{p}$-values less than 0.05 were considered as statistically significant factors.

Results: The magnitude of stunting, underweight and wasting were $48.7 \%, 21.7 \%$, and $8 \%$ respectively. Less than two times the daily intake of food $(\mathrm{AOR}=3.7,95 \% \mathrm{Cl}: 1.5,9.3)$. Being older child $(12-23$ months) (AOR=0.46, 95\% Cl, 0.22, 0.96) and low dietary diversity score (AOR=0.53, 95\% $\mathrm{Cl},(0.30,0.92)$ were significantly associated with undernutrition. Additionally, not having a hand washing facility in the toilet was a significant predictor for both wasting and underweight.

Conclusion: The prevalence of undernutrition among infants and young children was higher compared with the previous studies. Low dietary diversity score, lower meal frequency, being an older child, and absence of hand washing facility in the toilet were factors related to the nutritional status. Hence, interventions targeting community management of undernutrition might be appropriate to manage the problem. Efforts should also be intensified to reduce by focusing on identified determinants and the provision of nutrition education to mothers focusing on appropriate feeding practices to improve children's nutritional status in the study area.

\section{Background}

Now a day's undernutrition is the primary public health concern for many countries. Undernutrition can be defined as a lack of adequate energy, protein, and micronutrients to meet the basic requirements for body maintenance, growth, and development of children Adequate nutrition is essential in early childhood for healthy growth, proper organ formation and function, a strong immune system, and neurological and cognitive development [1, 2].

Undernutrition is one of the major causes of disability preventing children who survive from reaching their full development potential [3]. Besides, the undernourished status of the children locks them into a vicious cycle of recurring sickness, faltering growth, and diminished learning ability [4]. 
Even though a reduction has been made over the last decades in the burden of child undernutrition in developing countries, including Ethiopia the problem is still public health concern. Regardless of this success, children aged less than 2 years are still suffering from undernutrition in developing countries. Globally, more than about 178 million children in the world are found to be undernourished according to a recent (UNICEF / WHO) report [5]. The burden of undernutrition is much higher in South Asia compared to Africa and other parts of the world. The prevalence of underweight and stunting in South Asia has been recorded as $46 \%$ and $44 \%$, respectively [6]. Annually, 2.7 million or more than $45 \%$ of all child deaths are estimated to be associated with undernutrition more than $90 \%$ of these mortalities are reported in developing countries [7].

In Africa, $32 \%$ or 186 million children less than two years of age are stunted, and about $8 \%$ or 35 million are wasted. Stunting is associated with poor physical and cognitive development as well as low school performance in children and adolescents. The risk of mortality is higher among stunted children even though it is aggravated when stunting and wasting happened together[8]. Due to the intergenerational impact of chronic malnutrition, childhood stunting is correlated with future maternal undernutrition and a higher risk of death [9]. Premature delivery and giving birth to an underweight baby are commonly reported among undernourished mothers [10]. Under two years of age, children are the most susceptible age for undernutrition, because of their rapid growth and increased vulnerability to infectious disease.

Accordingly, low meal frequency and dietary diversity [11], and not taking prenatal iron supplementation [12], Poor wealth status, unavailability of a latrine, child age: 12-24 months, not receiving maternal postnatal vitamin-A supplementation and source of family food: own food production was significantly associated with higher odds of stunting [13]. Inappropriate age of complementary feeding initiation and bottle feeding, households with a low dietary diversity score [14] and households with the lowest wealth quintile, presence of diarrhea in the past 2 weeks [14], and higher child age [15] are identified as the determinants of stunting. Different studies also elicited the predictors of wasting, according to not breastfeeding during the previous day and diarrheal disease for wasting and underweight [13], being male, a high number of siblings [15] are associated with increased odds of wasting among children under-2 years.

Ethiopia is one of these countries undernutrition is an important public health problem; with a prevalence of stunting, wasting, and underweight of under two-year children were $23.2 \%, 16.6 \%$, and $12.5 \%$ respectively [16]. Although Ethiopia has made substantial achievements in providing for the wellbeing of its citizens, undernutrition in young children remains a major public health concern. Infant and young child feeding is a key area to improve child survival and promote healthy growth and development. About half of children aged six months to two years are not fed the minimum number of meals for their age, increasing their risk of stunting, wasting, and others. Ethiopia is being one of the countries with the highest child mortality rate, with undernutrition underlying $51 \%$ of all childhood deaths [17]. Currently, greater emphasis was given to nutrition in the training of health manpower and the use of health agents to monitor growth for effective prevention [18]. Despite increased attention to nutrition; nutritional deficiencies remain a devastating multifaceted problem affecting infants and young children in our 
country, especially in the Amhara region. The first 2 years of a child's life are particularly important, since optimal nutrition during this period lowers morbidity and mortality, reduces the risk of chronic disease, and fosters better development overall. Only some children receive nutritionally adequate and safe complementary foods; in many countries, less than a fourth of infants 6-23 months of age meet the criteria of dietary diversity and feeding frequency that are appropriate for their age.

Thus, the present study aimed to assess the nutritional status of children less than 2 years of age in a rural area of Sinan district to identify the factors associated with the occurrence of Undernutrition.

\section{Methods}

\section{Study area, design, and period}

A community-based cross-sectional study was conducted from March 2019 to April 25, 2019, in Sinan District, Northwest Ethiopia. Sinan District is located about $327 \mathrm{~km}$ far from Addis Ababa the capital city of Ethiopia and, $303 \mathrm{~km}$ away from Bahir Dar. The District is divided into 17 Keble's, two Keble's in the urban and 15 Keble's are rural. The total population was estimated as 120,000 , children aged $6-$ 23 months years old were 4251 . There are 5 health centers and 20 health posts in the District. The people in this area are engaged in different activities such as farming, and trade. The District is also known by its potato which is the main income-generating means for the community. The socio-cultural interaction is strong which shows working together during harvesting, good participation of celebration (wedding, mahiber, Edir, Ekub) together (Sinan District communication office) [19].

\section{Population}

The source population was all children aged 6-23 months who reside in Sinan District. The study population was all children aged 6-23 months living -in the selected Kebeles in Sinan District. All children from 6 months to 23 months old age who live in the selected Keble for at least 6 months were included in the study. However, children with a visible physical deformity like kyphosis, scoliosis were excluded from the study since it may under or overestimate anthropometric results.

\section{Sample Size And Sampling Procedure}

The required sample size was calculated by considering wasting, underweight, and stunting as exposure variables. Moreover, stunting was considered as an independent variable since it yielded a maximum sample size of which 374 . The sample size was computed on single population proportion formula by using: $95 \% \mathrm{Cl}$ (two-sided), 5\% margin of error, and 58.1\% proportion of stunting among children 6-23 months [14]. By considering 10\% for non-response rate a total sample size of 411 mother-child pairs was proposed. Of the 17 administrative clustered Keble's in the District, 6 clusters were selected by simple random sampling technique. A list of households with children aged between 6 to 23 months was 
obtained from the health post-EPI registration book. Then all the clustered households were included in the study. By using a cluster sampling technique a total of 431 infants and young children within the selected clustered Keble's was studied.

\section{Data Collection Tool And Procedure}

Data were collected through the home to home visits from the mothers or caregivers of the children using an interviewer-administered questionnaire. To maintain consistency, the questionnaire was first prepared in English and then translated to Amharic and then translated back to English. Six data collectors and three field supervisors were recruited for the study. The training was given to data collectors and supervisors. Length and weight measurements of children were taken using calibrated equipment and standardized techniques. The functionality of the equipment used to measure weight and length was checked each in each participant before measurement. Weight was measured to the nearest $10 \mathrm{~g}$ using a salter scale for less than 24 months old children. For all measurements, three readings were taken from each child, and the largest was recorded on the questionnaire. A standardized seven food group containing the Dietary Diversity Score (DDS) was used to assess the dietary intake of children [20]. A 24hour dietary recall method was used, accordingly, the mothers were asked to report the food items consumed by the child in the previous 24 -hours proceeding the days of the survey.

The household wealth index was computed using a composite indicator for urban and rural residents by considering properties such as selected household assets and the size of agricultural land. Using Principal Component Analysis (PCA), the factor scores were summed and ranked into poor, medium, and rich.

\section{Data processing and analysis}

Data were coded and entered into EPI-data version 4.2 statistical software and analysis was done by SPSS Version 25. Nutrition-related data (length and weight) were analyzed using the WHO Anthro software. The Z-scores of indices, Weight-for-Length Z-score (WLZ), WAZ, and Length-for-Age Z-score (LAZ) were calculated and compared using the WHO Multicenter Growth Reference Standard [21].

Bivariable Analysis was done individually for all independent variables with stunting, wasting, and underweight. Variables with a p-value of $<0.25$ in the Bivariable analysis were entered into a multivariable logistic regression analysis to identify the independent factors. Odds ratio a corresponding $95 \%$ Confidence interval $(\mathrm{Cl})$ were computed to assess the strength of the association. In the multivariable logistic regression analysis, variables with a $p$-value of $<0.05$ were considered as statistically significant. The fitness of the model was checked using the Hosmer and Lemeshow goodness of fit test.

\section{Operational Definitions}


Undernutrition was defined as the child having either of $\mathrm{H}$ /Age Z-score $<-2$, or W/Age Z-score $<-2$ or W/H Z-score $<-2$ SD [22, 23].

Underweight was defined as children having W/Age Z-score <-2 SD [22, 23].

Stunting was defined as children having $\mathrm{H} /$ Age Z-Score $<-2$ SD $[22,23]$.

Wasting was defined as children having W/H Z-score $<-2$ SD $[22,23]$.

\section{Minimum Dietary Diversity}

if a child has taken four food groups as the minimum acceptable dietary diversity, a child with a DDS of less than four was classified as poor dietary diversity; otherwise it was deemed to have good dietary diversity [20]

Food insecurity; Food insecurity is a condition in which people experienced limited or uncertain physical and economic access to safe, sufficient, and nutritious food to meet their dietary needs or food preferences for a productive, healthy, and active life [24]. It was assessed by using the household food insecurity access scale. Household food insecurity was measured using the Household Food Insecurity Access Scale (HFIAS) that was developed by the Food and Nutrition Technical Assistance (FANTA) project [24]. For the Household Food Insecurity Access Scale (HFIAS) measurement, each of the questions was asked with a recall period of four weeks (30 days). The respondent was first asked an occurrence question-that is, whether the condition in the question happened at all in the past four weeks (yes or no). If the respondent answers "yes" to an occurrence question, a frequency-of-occurrence question was asked to determine whether the condition happened rarely (once or twice), sometimes (three to ten times), or often (more than ten times) in the past four weeks.

\section{Wealth index}

The composite indicator of socio-economic status, which was computed by the application of principal component analysis (PCA). Initially, household asset data were prepared for analysis. Before the PCA, using frequency, important variables that can discriminate households were selected to reduce the number of variables. The binary variables were coded to 0 and 1 and categorical variable options were converted into binary variables and dummy variable was created as 0 and 1.After data preparation, variables were standardized to change variables into the same scale for comparison by subtracting the mean from each value and then dividing the standard deviation. Once standardized, the variables have a mean of 0 and a standard deviation of 1 . It was categorized as poor, medium, and rich.

\section{Poor socioeconomic status}

household which belonged to lower tercile of wealth index score.

\section{Medium socioeconomic status}


household which belonged to medium tercile of wealth index score.

\section{Rich socioeconomic status}

household which belonged to a higher tercile of wealth index score.

\section{Results}

\section{Socio-demographic characteristics}

From a total of 431 mother-child pairs to be included in the study, 427 of them participated with a response rate of $99.07 \%$. From these $263(61.6 \%)$ of the study children were male. The mean age of children was 13.42 months with an SD of $( \pm 4.90)$ months. Regarding their religion, almost all 422 $(98.8 \%)$ respondents were orthodox Christians. The majority of $393(92 \%)$ of children's mothers/ caregivers were married. One-tenth $48(11.2 \%)$ of mothers and $79(18.5 \%)$ of fathers had formal education. About $309(72.4 \%)$ of mothers were farmers and $237(55.5 \%)$ of fathers were farmers (Table 1$)$. 
Table 1

Socio-demographic and economic characteristics of the children and their parents, Sinan District, northwest Ethiopia, $2019(\mathrm{~N}=427)$

\begin{tabular}{|c|c|c|c|}
\hline Variables & & Frequency $(\mathrm{N})$ & Percent (\%) \\
\hline \multirow[t]{2}{*}{ Child age in months } & $6-11$ & 191 & 44.7 \\
\hline & $12-23$ & 236 & 55.3 \\
\hline \multirow[t]{2}{*}{ Sex of the child } & Male & 263 & 61.6 \\
\hline & Female & 164 & 38.4 \\
\hline \multirow[t]{2}{*}{ Marital status } & Married & 393 & 92 \\
\hline & Others* & 34 & 8 \\
\hline \multirow[t]{2}{*}{ Religion } & Orthodox & 422 & 98.8 \\
\hline & Others** & 5 & 1.2 \\
\hline \multirow[t]{4}{*}{ Maternal education } & No formal education & 379 & 88.7 \\
\hline & Primary & 17 & 4 \\
\hline & Secondary & 11 & 2.6 \\
\hline & College and above & 20 & 4.7 \\
\hline \multirow[t]{4}{*}{ Maternal occupation } & Farmer & 309 & 72.4 \\
\hline & House wife & 52 & 12.2 \\
\hline & Merchant & 43 & 10 \\
\hline & Other & 23 & 5.4 \\
\hline \multirow[t]{5}{*}{ Husband education } & Cannot read and write & 181 & 42.4 \\
\hline & Can read and write & 167 & 39.1 \\
\hline & Primary & 35 & 8.2 \\
\hline & Secondary & 12 & 2.8 \\
\hline & College and above & 32 & 7.5 \\
\hline \multirow[t]{5}{*}{ Husband occupation } & Farmer & 237 & 55.5 \\
\hline & Merchant & 50 & 11.7 \\
\hline & Daily laborer & 48 & 11.2 \\
\hline & Government employee & 55 & 12.9 \\
\hline & Others & 37 & 8.5 \\
\hline
\end{tabular}




\begin{tabular}{|llll|}
\hline Variables & & Frequency $(\mathrm{N})$ & Percent (\%) \\
\hline Head of household & Father & 372 & 87.1 \\
& Mother & 55 & 12.9 \\
\hline Family size & $\leq 4$ & 274 & 64.2 \\
& $5-7$ & 141 & 33 \\
& $>7$ & 12 & 2.8 \\
& 1st born & 132 & 30.9 \\
& 2nd born & 169 & 39.6 \\
& 3rd born & 69 & 16.2 \\
Wealth index & 4th and above & 57 & 13.3 \\
& Poor & 170 & 39.8 \\
& Medium & 153 & 35.8 \\
& Rich & 104 & 24.4 \\
\hline HFIAS & Food secure household & 347 & 81.3 \\
& Food insecure household & 80 & 18.7 \\
\hline
\end{tabular}

The majority of $368(92.7 \%)$ of the households in the study area used latrine But, 59 (13.8\%) of the households did not have a toilet facility. On the other hand, $248(41.9 \%)$ of the households had no household waste disposal site. This study revealed that $424(99.3 \%)$ of the mothers practiced washing their hands before feed their child. More than half 228 (53.4\%) of the households used public tape/standpipe water as a source of drinking water and one-fifth $86(20.1 \%)$ were using unprotected spring/ river as a source of drinking water. Conversely, one-fourth, $113(26.5 \%)$ were using protected well and spring water. Most 424 (99.3\%) of households reported that they washed their hands after the toilet and before eating. Only 3(0.7\%) reported that they did not wash their hands after toilet use (Table 2 ). 
Table 2

Water supply and hygienic conditions of the study participants $(n=427)$, Sinan Woreda, East Gojjam Zone, North West Ethiopia, 2019.

\begin{tabular}{|c|c|c|c|}
\hline Variables & & Frequency $(\mathrm{N})$ & Percent (\%) \\
\hline \multirow[t]{3}{*}{ Source of drinking water } & Tape water & 228 & 53.4 \\
\hline & Protected spring & 113 & 26.5 \\
\hline & Unprotected spring/river & 86 & 20.1 \\
\hline \multirow[t]{2}{*}{ Have latrine } & Present & 368 & 86.2 \\
\hline & Absent & 59 & 13.8 \\
\hline \multirow[t]{2}{*}{ Household use larine } & Yes & 355 & 83.1 \\
\hline & No & 13 & 3.1 \\
\hline \multirow[t]{3}{*}{ Kind of toilet } & Flush into a septic tank & 9 & 2.5 \\
\hline & Improved pit latrine & 187 & 50.8 \\
\hline & Unimproved pit latrine & 172 & 46.7 \\
\hline \multirow[t]{2}{*}{ Hand washing facility in the toilet } & Present & 296 & 75 \\
\hline & Absent & 98 & 25 \\
\hline \multirow[t]{2}{*}{ Hand washing material in the toilet } & Water only & 226 & 76.3 \\
\hline & Soap with water & 70 & 26.7 \\
\hline \multirow[t]{2}{*}{ Wash Your hand after the toilet } & Yes & 416 & 97.9 \\
\hline & No & 9 & 2.1 \\
\hline \multirow[t]{2}{*}{ Wash your hand before child feeding } & Yes & 424 & 99.3 \\
\hline & No & 3 & 0.7 \\
\hline \multirow[t]{3}{*}{ How to wash caregiver's hands } & Only with water & 211 & 49.4 \\
\hline & Soap with water sometimes & 120 & 28.1 \\
\hline & Soap with water always & 96 & 22.5 \\
\hline \multirow[t]{2}{*}{ The household waste disposal site } & Present & 179 & 58.1 \\
\hline & Absent & 248 & 41.9 \\
\hline
\end{tabular}

\section{Child caring and feeding practices of participants}

Among the study participants, $70(16.4 \%)$ of the child got minimum dietary diversity, which is greater than 4 food groups. More than half $281(65.8 \%)$ of the child took meals more than four times per day while, 
$27(6.3 \%)$ of the children took meals twice per day. Two-thirds $308(72.1 \%)$ of the caregivers had access to information about complementary feeding (Table 3).

Table 3

Household child feeding practice among study subjects $(\mathrm{n}=427)$, Sinan Woreda, East Gojjam Zone, Northwest Ethiopia, 2019.

\begin{tabular}{|c|c|c|c|}
\hline Variables & & Frequency(N) & Percent\% \\
\hline \multirow[t]{3}{*}{ Frequency of meals } & Twice Three times & 27 & 6.3 \\
\hline & \multirow[t]{2}{*}{ Four times and above } & 119 & 27.9 \\
\hline & & 281 & 65.8 \\
\hline \multirow[t]{2}{*}{ Dietary diversity score } & $<4$ food groups & 357 & 83.6 \\
\hline & $\geq 4$ food groups & 70 & 16.4 \\
\hline \multirow{2}{*}{$\begin{array}{l}\text { Access to information on complementary } \\
\text { feeding from different sources }\end{array}$} & Yes & 308 & 72.1 \\
\hline & No & 119 & 27.9 \\
\hline \multirow[t]{2}{*}{ Give Abish (fenugreek) to your child } & Yes & 365 & 14.5 \\
\hline & No & 62 & 85.5 \\
\hline \multirow[t]{3}{*}{ With whom your child is eating mostly } & \multirow{2}{*}{$\begin{array}{l}\text { Separately } \\
\text { Father/mother/caregiver }\end{array}$} & 375 & 87.1 \\
\hline & & 15 & 3.5 \\
\hline & Older siblings & 40 & 9.4 \\
\hline \multirow[t]{3}{*}{ Meals the child usually eat mostly } & Breakfast & 238 & 55 \\
\hline & Lunch & 144 & 33.7 \\
\hline & Dinner & 48 & 11.2 \\
\hline \multirow[t]{3}{*}{ Vaccination status } & Completely vaccinated & 415 & $97.2 \%$ \\
\hline & Incomplete vaccination & 4 & 0.9 \\
\hline & $\begin{array}{l}\text { Vaccinated According to } \\
\text { his/her age }\end{array}$ & 8 & 1.8 \\
\hline \multirow[t]{2}{*}{ Diarrhea in the last two weeks } & Yes & 46 & 89.2 \\
\hline & No & 381 & 10.8 \\
\hline \multirow[t]{2}{*}{ Fever in the last two Weeks } & Yes & 44 & 89.7 \\
\hline & No & 383 & 10.3 \\
\hline
\end{tabular}

\section{Factors associated with stunting of participants}


The Bivariable logistic regression analysis showed that the frequency of meal taken by children, being second-order birth, being from a food-insecure household, the occurrence of diarrhea, presence of fever, head of household, and dietary diversity score less than four food groups was found to be associated with stunting. In the multivariable logistic regression, having adequate dietary diversity score and frequency of meal taken by children were significantly associated with stunting.

Children who get food less than three times per day were more than three and half times more likely to experience stunting as compared to their counterparts, [AOR $=3.5 ; 95 \% \mathrm{Cl}, 1.5-9.3]$. Having adequate dietary diversity score was $47 \%$ less risk of stunting as compared to their counterparts (AOR $=0.53,95 \%$ $\mathrm{Cl}, 0.30-0.92)$ Table 4) 
Table 4

Factors associated with stunting on selected variables among children 6-23 months old in Sinan District, East Gojjam Zone, North West Ethiopia, $2019(\mathrm{n}=$ 427)

\begin{tabular}{|c|c|c|c|c|}
\hline \multirow[t]{2}{*}{ Variables } & \multicolumn{2}{|c|}{ Stunting } & \multirow[t]{2}{*}{$\operatorname{coR}(95 \% \mathrm{Cl})$} & \multirow[t]{2}{*}{ AOR Cl 95\% } \\
\hline & Yes & No & & \\
\hline \multicolumn{5}{|l|}{ Food security } \\
\hline Food secure & 162 & 185 & 1 & 1 \\
\hline Food insecure & 46 & 34 & $1.5(0.9,2.5)$ & $1.7(1,2.9)$ \\
\hline \multicolumn{5}{|l|}{ Dietary diversity score } \\
\hline$<4$ food groups & 166 & 191 & 1 & 1 \\
\hline$\geq 4$ food groups & 42 & 28 & $0.57(0.3,0.9)$ & $0.53(0.30,0.92)$ \\
\hline \multicolumn{5}{|l|}{ Birth order of index child } \\
\hline 1 st & 56 & 76 & 1 & 1 \\
\hline 2nd & 92 & 77 & $1.6(1.02,2.5)$ & $1.4(0.92,2.3)$ \\
\hline $3 r d$ & 27 & 42 & $0.87(0.47,1.5)$ & $0.78(0.42,1.4)$ \\
\hline 4th and above & 33 & 24 & $1.8(0.9,3.5)$ & $1.4(0.75,2.8)$ \\
\hline \multicolumn{5}{|l|}{ Fever } \\
\hline No & 182 & 201 & $1.5(0.85,3.00)$ & $1.7(0.92,3.4)$ \\
\hline Yes & 26 & 18 & 1 & 1 \\
\hline \multicolumn{5}{|l|}{ Diarrhea last two week } \\
\hline No & 180 & 201 & 1 & 1 \\
\hline Yes & 28 & 18 & $1.7(0.92,3.2)$ & $1.7(0.88,3.4)$ \\
\hline \multicolumn{5}{|l|}{ Meal frequency } \\
\hline Twice & 20 & 7 & $3.1(1.2,7.6)$ & $3.7(1.5,9.3)$ \\
\hline Three times & 54 & 65 & $0.9(0.5,1.4)$ & $1.02(0.6,1.6)$ \\
\hline Four times and above & 134 & 147 & 1.00 & 1 \\
\hline \multicolumn{5}{|l|}{ Head of household } \\
\hline Father & 186 & 186 & 1 & 1 \\
\hline Mother & 33 & 22 & $0.66(0.37,1.1)$ & $0.66(.38,1.34)$ \\
\hline
\end{tabular}




\section{Factors Associated With Wasting Of Participants}

In Bivariable logistic regression analysis being older age (12-23) months, low dietary diversity score and absence of handwashing facility in the toilet were significantly associated with wasting whereas multivariable analysis, being older age ( $\mathrm{AOR}=0.46,95 \% \mathrm{Cl}, 0.22-0.96)$, and absence of Handwashing facility in the toilet $(A O R=2.5,95 \% \mathrm{Cl},(1.1-5.9)$ were significantly associated with wasting.

In this study, children found in the age range of 12-23 months were $54 \%$ less risk of being wasted as compared to those children in the age range of 6-11 months ( $\mathrm{AOR}=0.46,95 \% \mathrm{Cl}, 0.22-0.96)$. Children living in households who had no handwashing facility in the toilet were 3.7 times more at risk of being wasted than children of households who had a handwashing facility in the toilet $(\mathrm{AOR}=3.7,95 \% \mathrm{Cl},(1.7-$ 7.8) (Table 5).

Table 5

Factors associated with wasting of undernutrition on selected variables among children 6-23 months old in Sinan Woreda, East Gojjam Zone, North West Ethiopia, $2019(n=427)$

\begin{tabular}{|lllll|}
\hline Variables & Wasting & COR(95\% Cl) & AOR(95\%Cl) \\
\hline & Yes & No & & \\
\hline Age & & & & 1 \\
\hline $6-11$ & 21 & 170 & 1 & $0.46(0.22,0.96)$ \\
\hline $12-23$ & 13 & 223 & $0.47(0.23,0.96)$ & \\
\hline Dietary diversity score & & & & $2.7(0.6,11)$ \\
\hline$<4$ food groups & 32 & 325 & $3.3(0.7,14)$ & 1 \\
\hline$\geq 4$ food groups & 2 & 68 & 1 & 1 \\
\hline Hand washing facility toilet & & & & $3.7(1.7,7.8)$ \\
\hline Yes & 18 & 311 & 1 & \\
\hline No & 16 & 82 & $3.3(0.16,6.8)$ & \\
\hline
\end{tabular}

\section{Factors Associated With Underweight Of Participants}

Not having a handwashing facility in the toilet, being a younger age child and low meal frequency was significantly associated with underweight in Bivariable analysis. Multivariable logistic regressions showed that children living in households without handwashing facility in the toilet were 2.6 times more likely to be underweight than children living in households of having handwashing facility in the toilet $(\mathrm{AOR}=2.6,95 \% \mathrm{Cl},(1.5-4.3)$ (Table 6) 
Table 6

Factors associated with underweight on selected variables among children 6-23 months old in Sinan District, East Gojjam Zone, North West Ethiopia, $2019(n=427)$

\begin{tabular}{|c|c|c|c|c|}
\hline \multirow[t]{2}{*}{ Variables } & \multicolumn{2}{|c|}{ Underweight } & \multirow[t]{2}{*}{$\operatorname{coR}(95 \% \mathrm{Cl})$} & \multirow[t]{2}{*}{ AOR $(95 \% \mathrm{Cl})$} \\
\hline & Yes & No & & \\
\hline \multicolumn{5}{|l|}{ Age } \\
\hline $6-11$ & 49 & 142 & 1 & 1 \\
\hline $12-23$ & 44 & 192 & $0.66(0.41-1.0)$ & $0.64(0.40-1.02)$ \\
\hline \multicolumn{5}{|l|}{ Meal frequency } \\
\hline Twice & 7 & 20 & $1.4(0.56-3.4)$ & $0.92(0.34-2.4)$ \\
\hline Three times & 30 & 89 & $1.3(0.81-2.2)$ & $1.05(0.60-1.83)$ \\
\hline More than Four times & 56 & 225 & 1 & 1 \\
\hline \multicolumn{5}{|c|}{ Hand Washing facility in the toilet } \\
\hline Absent & 35 & 63 & $2.5(1.5-4.2)$ & $2.6(1.5-4.3)$ \\
\hline Present & 58 & 271 & 1 & 1 \\
\hline
\end{tabular}

\section{Discussion}

In this study, the prevalence of undernutrition among infants and young children is found to be high with the prevalence of stunting $48.7 \%$, underweight $21.7 \%$, and wasting $8 \%$ respectively. The high prevalence of stunting in this study suggests the severe public health significance of the problem. The prevalence of stunting in this study is higher than previous studies conducted in Ethiopia Demographic and Health Survey 23.2\%[25]. This finding also showed that a higher percentage of stunting as compared with studies conducted in Angola 32\% [26], Mali 28.4\% [15], Kamba Woreda 27.8\% [27] but, lower than studies done in Gondar 58.1\% [14] and Somali region 56.1\% [13]. The differences might be due to variation in time, sample size, study period, socio-economic characteristics of study participants, and a difference in infant and young child feeding practices.

Moreover, this study revealed that undernutrition in the study area is a critical public health problem according to the WHO cut-off point [9]. The finding of underweight obtained in this study is in line with reports of other developing countries, such as Mali, 23.9\% [15], and the Central Republic of Africa (20.2\%). However, this finding is higher than studies reported from the rural part of Angola 15.1\% [26], Somali region Awbare 10\% [13], the national report of EDHS 2016 12.5\%[25] and Benna Tsemay Woreda, 15.3\% [27]. This might be due to the difference in the sample size and study period between the current and previous studies. Moreover, the difference observed underweight might be due to the difference in the socio-economic, feeding, lifestyle, and socio-demographic characteristics of the study participants. 
The prevalence of wasting documented in this study is comparable with studies done in Benny Tsemay Kemba, 9.9\% [27], and the Somali region 10\% [13]. On the other hand, this finding is lower than studies conducted in Gondar (17\%) [14], Ethiopia Demographic Health Survey 16.6\% [25], and Mali (13.9\%) [15]. The variation might be due to the difference in sample size, study settings, frequency of feeding, socioeconomic status, and food security. A similar study was reported But, the finding of this study was lower compared to a study done in Angola [19].

This study also focused on identifying the factors that have an association with the undernutrition status of infants and young children aged 6-23 months in the Sinan district. This depicted that low dietary diversity score and low daily meal frequency were identified as a significant factor for the occurrence of stunting. This finding was supported by a study conducted in Egypt [28] and Mali [15]. The similarity might be due to the similarity in socio-economic characteristics particularly on community awareness towards initiation of complementary feeding and diversified food during early childhood ages. Information on complementary feeding is important for starting complementary food based on the recommended date.

In this study child, 6-11 months old age were statically associated factors with wasting. The probable reason might be because of late initiation and inappropriate complementary feeding practice to meet nutritional demand were practiced in the study area. These factors were not reported as a factor of wasting in the previous study rather being an older child was a significant factor for being stunted in the previous literature.

In this study absence of a hand washing facility in the toilet was also identified as a factor for both underweight and wasting. In the previous research, this factor was not reported as a significant factor in different kinds of literature.

\section{Limitation Of The Study}

This study recognized the following limitations:

There may be a recall bias in 24 hours recall dietary assessment and measurement bias during data collection time that might affect the study findings. The cross-sectional study may not enable to determine causal relationships.

\section{Conclusion}

The result of this study described that the prevalence of stunting, underweight, and wasting among infants and young children aged 6-24 months was relatively high. This showed that undernutrition is still a major public health problem among infants and young children.

A low frequency of daily meal, Lack of adequate dietary diversity score, being younger age of children (611 months) and, Absence of hand washing facility in the toilet was found to be significantly associated 
with undernutrition.

\section{Recommendations}

Based on the finding of this study the following recommendations were forwarded to the concerned bodies. Promote nutrition counseling on appropriate feeding practices, nutrition counseling about the frequency and diversity of diet to all children especially 6-23 months of age, Emphasis should be given for children aged 6-11 months to start adequate complementary foods early in addition to breastfeeding. Additionally, Education should be given to the community to have a hand washing facility in the toilet.

\section{Abbreviations}

DDS: Dietary Diversity Score, EDHS: Ethiopia Demographic Health Survey, WAZ: Weight for Age Z score, HAZ: Height for Age Z score, WHZ: Weight for Height Z score, UNICEF: United Nation Children's Fund, WHO: World Health Organization.

\section{Declarations}

Ethical approval was obtained from Debre Markos University, College of Health Sciences. An official letter was also written along with the ethical clearance and submitted to Sinan District Administration Health Office. The autonomy of the participants was respected. The purposes and importance of the study were explained at all levels to the caregivers and oral informed consent was secured from each child caregiver. Caregivers selected for the study were on their verbal consents; caregivers who were not voluntary to be interviewed were informed to do so without any restriction. Children's who were found malnourished during the anthropometric measurement were linked to the nearby health facilities for nutritional counseling and treatment.

\section{Consent for publication}

Not applicable

\section{Availability of data and material}

The data sets used and/or analyzed during the current study are available from the corresponding author on reasonable request.

\section{Competing interests}

The authors have declared that they have no competing interests. 
Authors' contribution

MG: conception of the research idea, study design, data collection, analysis and interpretation, and manuscript write-up. GK, NA, YS, EF data collection, analysis, interpretation and manuscript write-up. All authors have read and approved the final manuscript.

\section{Funding}

Not applicable

\section{Acknowledgment}

The authors would like to acknowledge the Debre Markos University, College of Medicine and Health Sciences for giving the opportunity to do this research. The authors also extend their special thanks for both data collectors and supervisors. The authors also would like to acknowledge Mr. Nurilign Abebe (BSc, MPH, Assistant professor), Mr. Gemechu Kumera (BSc, MSc) for their constructive comments and suggestions.

\section{References}

1. UNICEF. Improving Child Nutrition: The achievable imperative for global progress. In. New York, NY 10017, USA; 2013.

2. Bank UWW: Group: Levels and Trends in Child Malnutrition, Joint child malnutrition estimates 2018 (UNICEF-WHO-WB). http:// datatopics. worldbank.org/child-malnutrition/.. In.; 2018.

3. Health ACloP.. Community-Based Sub-Component of Ethiopian National Nutrition Program Baseline Survey Report.. In.; 2016.

4. Caulfield LE, dOM, Blössner M, Black RE. Undernutrition as an underlying cause of child deaths associated with diarrhea, pneumonia, malaria, and measles. Am J Clin Nutr 2014.

5. World Health Organization. Essential nutrition actions: improving maternal, newborn, infant, and young child health and nutrition. Geneva: WHO; 2013 [accessed in 201911 Jan]. Available from: http://apps.who.int/iris/bitstream/10665/84409/1/9789241505550_eng.pdf. In.

6. WHO. Essential nutrition actions: improving maternal, newborn, infant, and young child health and nutrition. Geneva: WHO; Available from 
http://apps.who.int/iris/bitstream/10665/84409/1/9789241505550_eng.pdf. In.; 2019.

7. WHO. World Health Organization [internet]. Nutrition Landscape Information System (NLiS). Geneva: Nutrition; 2016 [accessed in 201914 Jan]. Available from: http://www.who.int/nutrition/nlis/en. In.; 2016.

8. McDonald CM, Olofin I, Flaxman S, Fawzi WW, Spiegelman D, Caulfield LE, Black RE, Ezzati M, Danaei G, Study ftNIM. The effect of multiple anthropometric deficits on child mortality: a metaanalysis of individual data in 10 prospective studies from developing countries. The American Journal of Clinical Nutrition. 2013;97(4):896-901.

9. World Health O: WHO child growth standards: length/height-for-age, weight-for-age, weight-for-length, weight-for-height, and body mass index-for-age: methods and development. In. Geneva: World Health Organization; 2006.

10. Jawaid SA. The Global Action Report on Preterm Birth. Pulse International 2012, 13.

11. Agedew E, Chane T. Prevalence of Stunting among Children Aged 6-23 Months in Kemba Woreda, Southern Ethiopia: A Community Based Cross-Sectional Study. Advances in Public Health. 2015;2015:164670.

12. Mzumara B, Bwembya $P$, Halwiindi $H$, Mugode R, Banda J. Factors associated with stunting among children below five years of age in Zambia: evidence from the 2014 Zambia demographic and health survey. BMC Nutrition. 2018;4(1):51.

13. Fekadu Y, Mesfin A, Haile D, Stoecker BJ. Factors associated with nutritional status of infants and young children in Somali Region, Ethiopia: a cross-sectional study. BMC Public Health. 2015;15(1):846.

14. Derso T, Tariku A, Bikes GA, Wassie MM. Stunting, wasting and associated factors among children aged 6-24 months in Dabat health and demographic surveillance system site: A community based cross-sectional study in Ethiopia. BMC Pediatrics. 2017;17(1):96.

15. Makamto Sobgui C, Kamedjie Fezeu L, Diawara F, Diarra H, Afari-Sefa V, Tenkouano A. Predictors of poor nutritional status among children aged 6-24 months in agricultural regions of Mali: a crosssectional study. BMC Nutrition. 2018;4(1):18.

16. EDHS E. demographic and health survey 2016: key indicators report. The DHS Program ICF 2016.

17. S C: Decentralization and scale-up of outpatient management of SAM in Ethiopia 39-40 (2011).

18. Bekele A. E: Malnutrition in women and children in Ethiopia. Addis Abab. 1991.

19. Temesgen $\mathrm{H}$, Yeneabat $\mathrm{T}$, Teshome $\mathrm{M}$. Dietary diversity and associated factors among children aged 6-23 months in Sinan Woreda, Northwest Ethiopia: a cross-sectional study. BMC nutrition. 2018;4(1):5.

20. Organization WH, UNICEF: Indicators for assessing infant and young child feeding practices part 3 : country profiles [periódico na internet]. 2010;[citado 2016 Jan 19]. In.

21. WHO. WHO child growth standards: length/height-for-age, weight-for-age, weight-for-length, weightfor-height and body mass index-for-age: methods and development. Geneva: WHO Press; 2006. 
22. World Health Organization. WHO Child Growth Standards available at http://www.who.int/childgrowth/standards/Technical_report.pdf. 2006.

23. Turck D, Michaelsen KF, Shamir R, Braegger C, Campoy C, Colomb V, Decsi T, Domellöf M, Fewtrell M, Kolacek S: World health organization 2006 child growth standards and 2007 growth reference charts: a discussion paper by the committee on nutrition of the European society for pediatric gastroenterology, hepatology, and nutrition. J Pediatr Gastroenterol Nutr 2013, 57(2):258-264.

24. Food and Agriculture Organization of the United Nations IFAD U, World Food Program, World Health Organization.: THE STATE OF FOOD SECURITY AND NUTRITION IN THE WORLD. BUILDING RESILIENCE FOR PEACE AND FOOD SECURITY, Rome, FAO.. In.; 2017.

25. Demographic EE: Health Survey. Key Indicators Report. Central Statistical Agency Addis Ababa, Ethiopia. Rockville: The DHS Program, ICF; 2016. In.; 2018.

26. Humbwavali JB, Giugliani C, Nunes LN, Dalcastagnê SV, Duncan BB. Malnutrition and its associated factors: a cross-sectional study with children under 2 years in a suburban area in Angola. BMC Public Health. 2019;19(1):220.

27. Agedew E, Chane T: Prevalence of stunting among children aged 6-23 months in Kemba Woreda, Southern Ethiopia: a community based cross-sectional study. Advances in Public Health 2015, 2015.

28. Maged M, kamal n: Prevalence of Stunting, Overweight, and Obesity among Egyptian Primary School Children in Behera Governorate. 2019, 9:84-93.

\section{Figures}



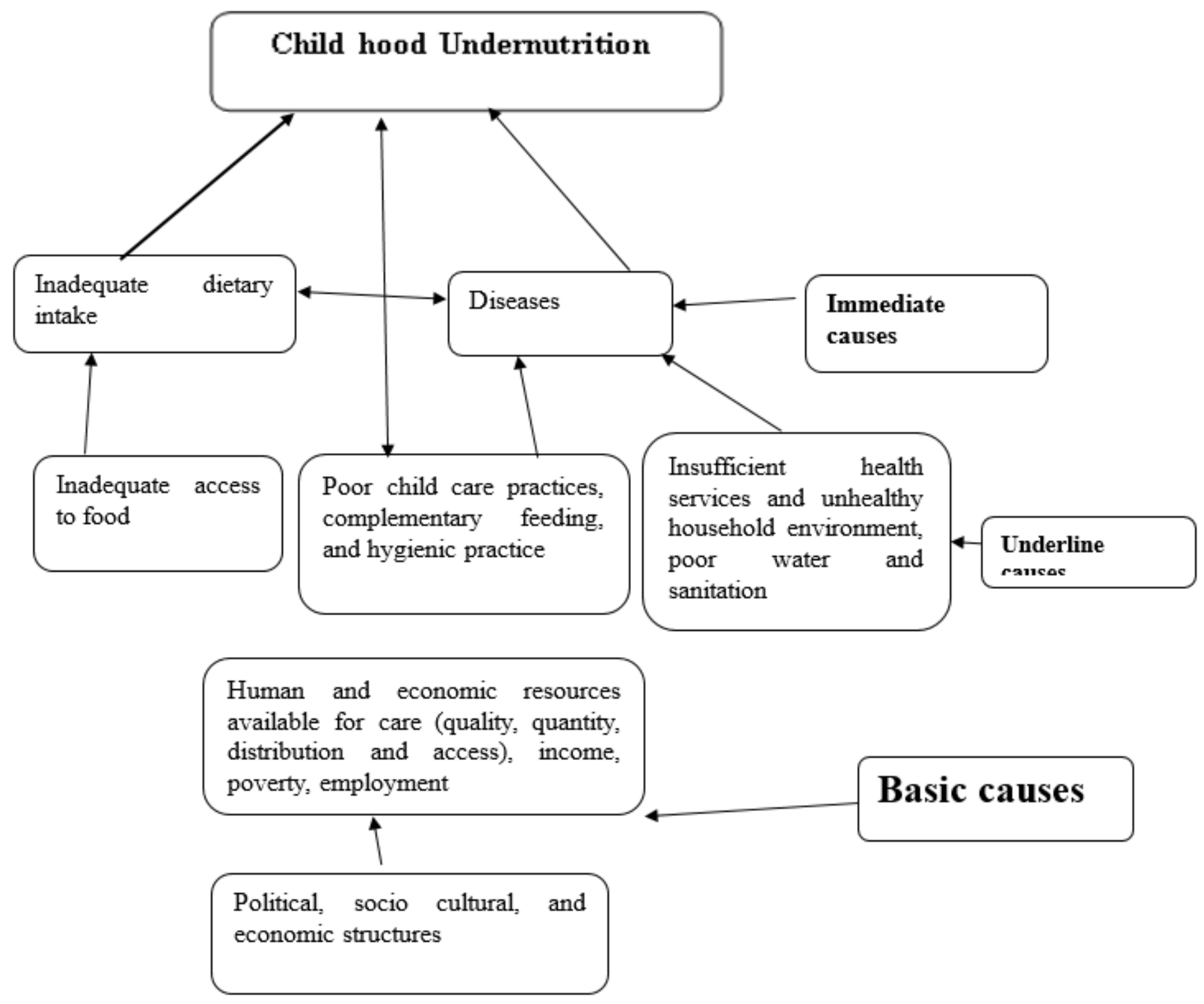

Figure 1

Conceptual framework of factors associated with malnutrition (adopted from UNICEF 2010) 


\section{Sinan district Total Keble $=19$}

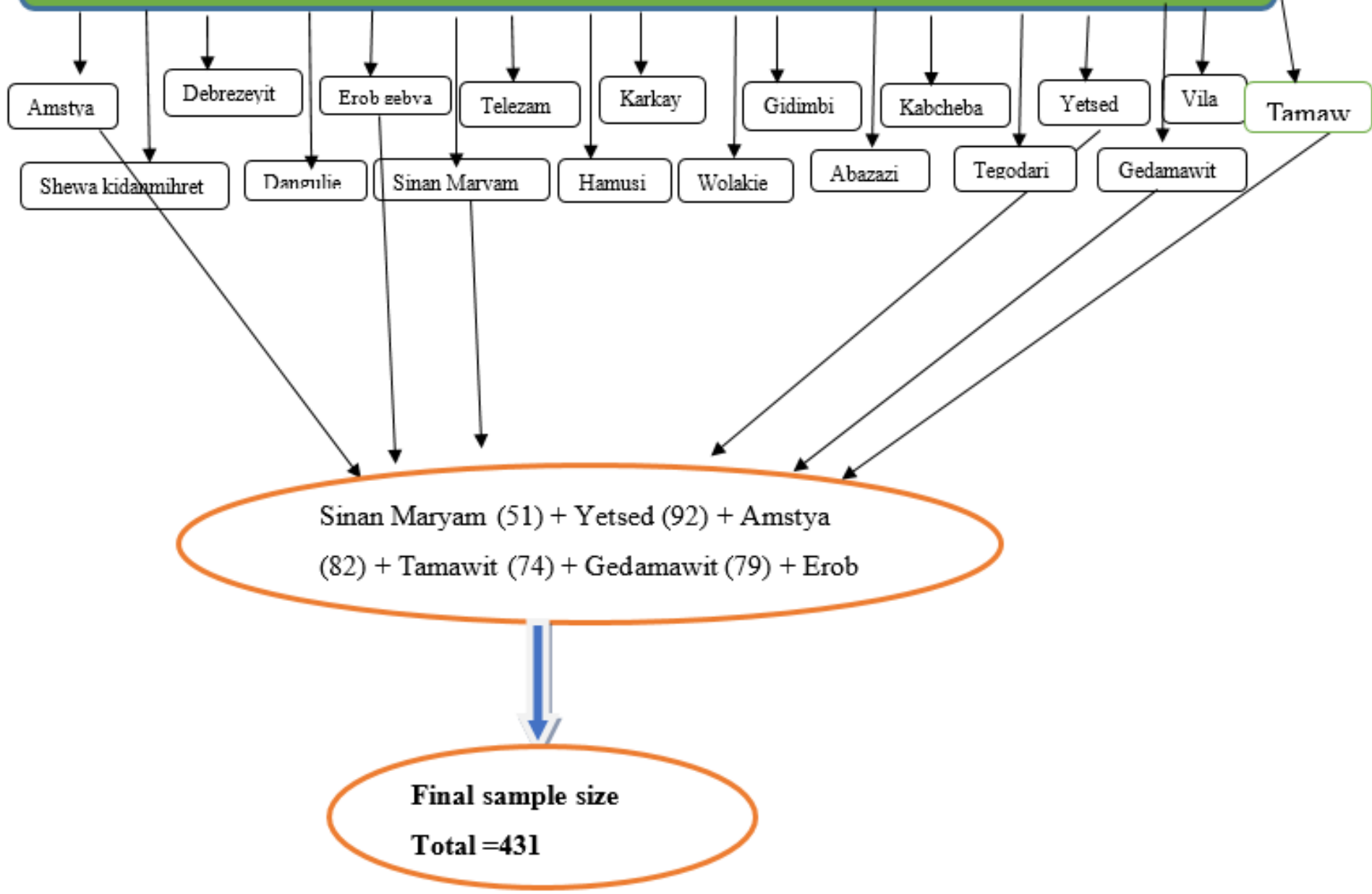

Figure 2

Schematic presentation of sampling procedure in Sinan District, North West Ethiopia, 2019. 


\section{Weight for length}

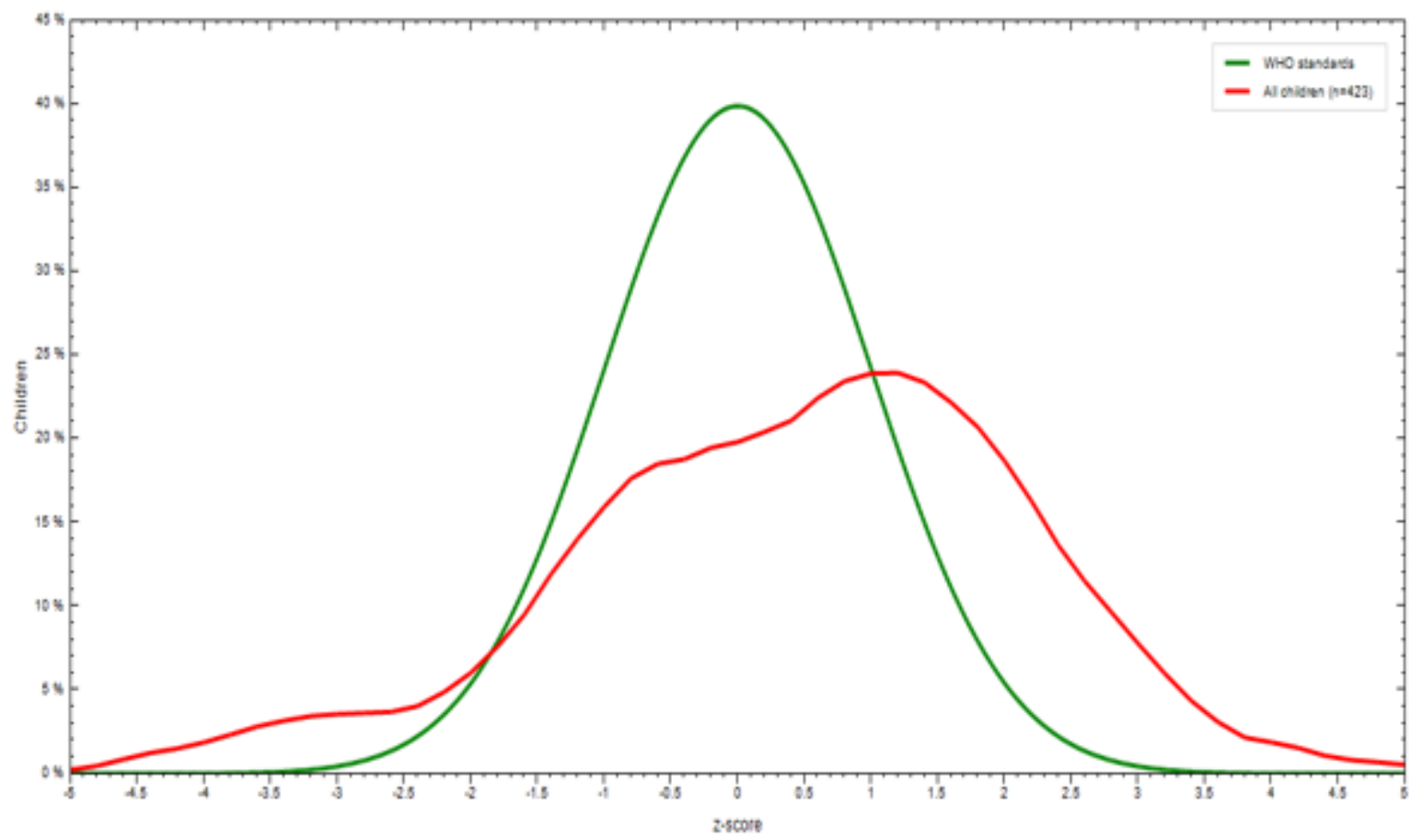

Figure 3

Distribution of wasting among children 6-23 months old in Sinan District, compared with WHOs standard population Sinan District, East Gojjam Zone, North West Ethiopia, $2019(\mathrm{n}=427)$.

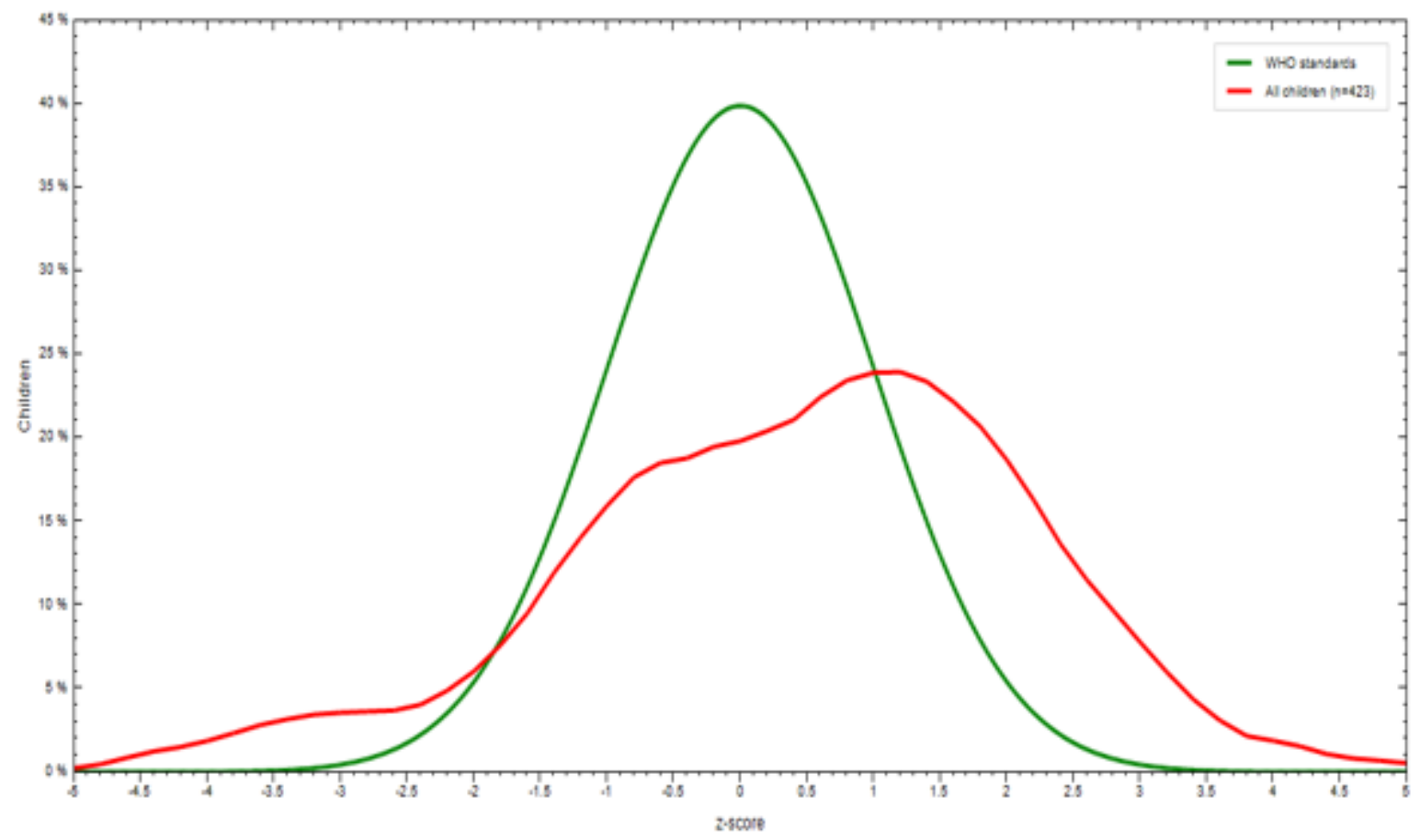

Figure 4 
Distribution of wasting among children 6-23 months old in Sinan District, compared with WHOs standard population Sinan District, East Gojjam Zone, North West Ethiopia, $2019(\mathrm{n}=427)$.

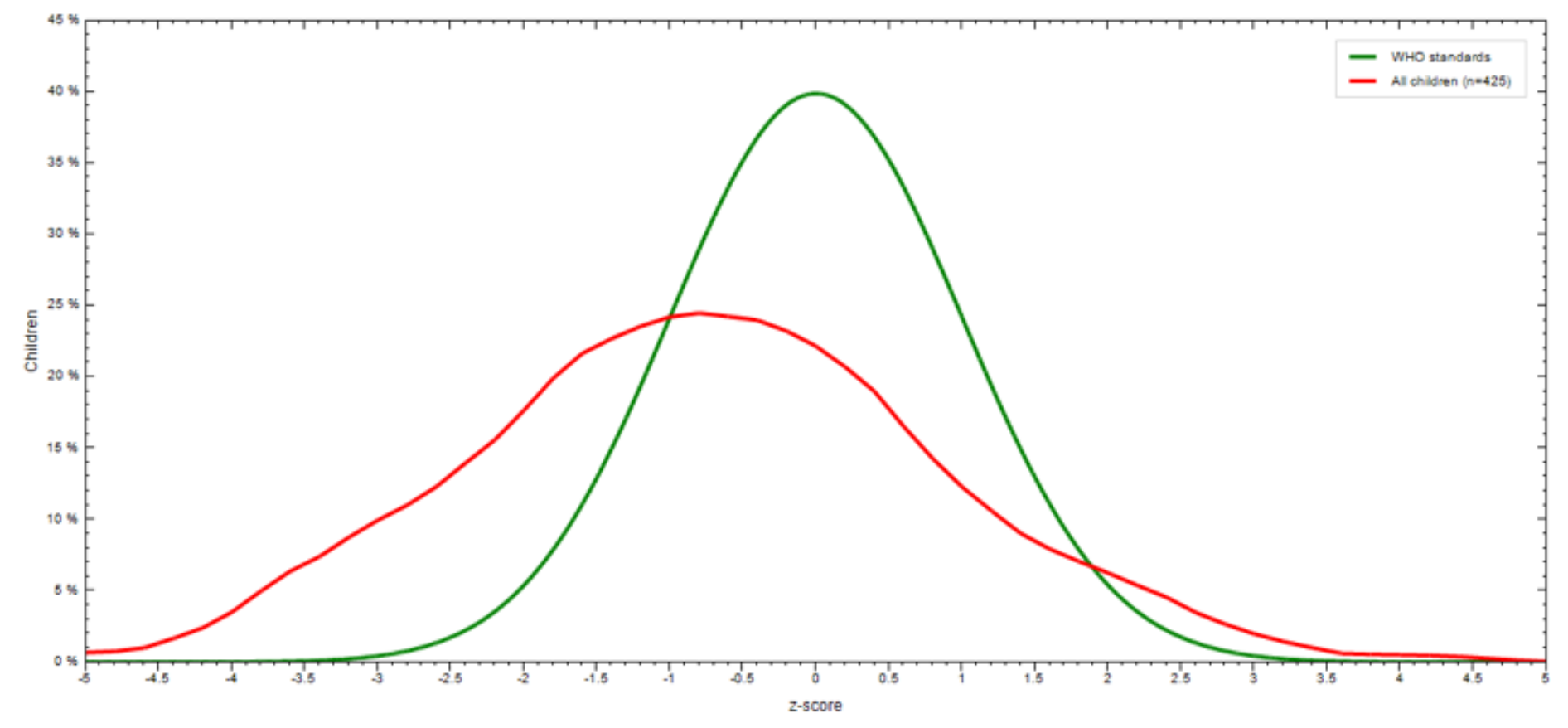

Figure 5

Distribution of underweight among children 6-23 months old in Sinan District, compared with WHOs standard population Sinan Woreda, East Gojjam Zone, North West Ethiopia, $2019(n=427)$.

\section{Supplementary Files}

This is a list of supplementary files associated with this preprint. Click to download.

- ANNEX.docx 